\title{
Factores relacionados con la intención de desertar en estudiantes de enfermería
}

\author{
Factors relating to nurse students intending to drop out \\ Fatores relacionados com a intenção de desertar em estudantes de enfermagem \\ Carolina Vargas-Porras* \\ Dora Inés Parra** \\ Zayne Milena Roa-Díaz ${ }^{* * *}$
}

\begin{abstract}
Autor de correspondencia
* * Enfermera. Magíster en Enfermería Materno Perinatal. Doctoranda en Enfermería y Salud. Profesora Asociada Escuela de Enfermería, Universidad Industrial de Santander. E-mail: cvargasporras@yahoo. com Orcid: (iD https://orcid.org/0000-00025996-8732 Bucaramanga, Colombia.

** Enfermera. Especialista en Alta Gerencia $y$ en atención de desastres y emergencias. Magíster en Enfermería énfasis en servicios de salud. Doctoranda en Enfermería. Profesora Asociada Escuela de Enfermería, Universidad Industrial de Santander. E-mail: doraiparra@yahoo.com Orcid: (ID http://orcid. org/0000-0003-1527-973X Bucaramanga, Colombia.

*** Enfermera. Magíster en Epidemiología. Profesora Escuela de Enfermería, Universidad Industrial de Santander. Bucaramanga, Colombia. E-mail: zaynemilena6@gmail. com. Orcid: (D) https://orcid.org/0000-00020847-0969 Bucaramanga, Colombia.
\end{abstract}

Este es un artículo bajo la licencia CC BY

(https://creativecommons.org/

licenses/by/4.0/) @) (1)

\section{Resumen}

Objetivo: Determinar los factores relacionados con la intención de desertar en estudiantes de enfermería de una universidad pública en Colombia. Materiales y Métodos: estudio de corte transversal analítico. La muestra correspondió a 162 estudiantes de los diferentes niveles académicos. Para identificar los factores de riesgo se creó un cuestionario con 54 ítems que se sometió a validez facial, e incluyó una pregunta para establecer la intención de desertar de la carrera en el último mes. Se realizó un análisis descriptivo y se construyó un modelo de regresión logística que identificó los factores relacionados con la presencia de intención de desertar de la carrera. El análisis estadístico se realizó en el programa Stata v12 software. Resultados: Promedio de edad de los participantes fue 21 años, el $85 \%$ pertenecen al género femenino; el $20 \%(\mathrm{n}=32)$ declaró haber considerado desertar del programa de enfermería en el último mes, de estos $78 \%$ (25/32) cursaban sexto o niveles inferiores. Los factores de riesgo para la intención de desertar fueron: síntomas depresivos de ansiedad y depresión; falta de interés en las asignaturas del programa relacionado con ausencia de identidad vocacional; relación regular con los profesores y pertenecer a estrato socioeconómico bajo. Conclusiones: El interés por las asignaturas y la relación con los profesores están relacionados con la intención de desertar, así como el componente de salud mental y el estrato socioeconómico. Se requieren estudios longitudinales para corroborar estos hallazgos.

Palabras Clave: factores de riesgo, deserción, educación superior.

Abstract

Objective: Determine factors related to the intention of dropping out of nursing students in public universities in Colombia. Materials and Methods: Cross-sectional analytic

‡ Este artículo es producto del proyecto de investigación titulado "factores de riesgo para la deserción estudiantil en estudiantes del programa de enfermería de la universidad" con código FS201604, aprobado por la Vicerrectoría de Investigación y Extensión de la Universidad, Colombia. 
study. The sample corresponded to 162 students of different academic levels. To identify the risk factors a questionnaire with 54 items was created which was subject to facial validity and included a question to establish the intention of dropping out in the last month. A descriptive analysis was made, and a model of logistic regression was made to identify the factors related to the intention of dropping out. The statistical analysis was made with the program software Statav12. Results: The average age of the participants was $21,85 \%$ were females; $20 \%(n=32)$ declared having thought about dropping out from the nursing program in the last month, $78 \%$ of these $(25 / 32)$ were in the sixth semester or earlier in the career. The risk factors for dropping out were: depressive symptoms of anxiety and depression; lack of interest in the classes offered by the program related to absence of vocational identity; regular interaction with the professors and belonging to a low socioeconomic population. Conclusions: The interest in classes and the interaction with the professors are related with the intention of dropping out, as well as the mental health component and the socioeconomic status. Longitudinal studies are required to corroborate these findings.

Keywords: risk factors, dropout, higher education.

\section{Resumo}

Objetivo: Determinar os fatores relacionados com a intenção de desertar em estudantes de enfermagem de uma universidade pública na Colômbia. Materiais e Métodos: estudo de corte transversal analítico. A amostra correspondeu a 162 estudantes dos diferentes níveis acadêmicos. Para identificar os fatores de risco se criou um questionário com 54 itens que se someteu a validade facial, e incluiu uma pergunta para estabelecer a intenção de desertar do curso no último mês. Realizou-se uma análise descritiva e se construiu um modelo de regressão logística que identificou os fatores relacionados com a presença de intenção de desertar do curso de enfermagem. A análise estadística se realizou no programa Stata v12 software. Resultados: A média de idade dos participantes foi de 21 anos, o 85\% pertenceram ao género feminino; o 20\% $(n=32)$ declarou haver considerado desertar do programa de enfermagem no último mês, destes 78\% (25/32) cursavam sexto ou níveis inferiores. Os fatores de risco para a intenção de desertar foram: sintomas depressivos de ansiedade e depressão; falta de interesse nas disciplinas do programa relacionado com ausência de identidade vocacional; relacionamento regular com os professores e pertencer a baixo estrato socioeconómico. Conclusões: O interesse pelas disciplinas e o relacionamento com os professores estão relacionados com a intenção de desertar, assim como, o componente de saúde mental e o estrato socioeconômico. Requerem-se estudos longitudinais para corroborar estas descobertas.

Palavras-chave: educação superior, deserção, fatores de risco.

\section{Introducción}

La deserción es un problema que enfrentan los diferentes sistemas educativos de las instituciones de educación superior en todo el mundo y aunque en algunos se han realizado esfuerzos para mejorar la situación, ésta sigue vigente. A ella se te asocian varios aspectos, que van desde los individuales hasta los institucionales y del propio Estado. Para los individuos es la causante de grandes frustraciones (1) y para la sociedad indicador de subdesarrollo, debido a las implicaciones que tiene en el crecimiento de un país.

De acuerdo al Observatorio de Educación Superior (2), la deserción "hace referencia al abandono prematuro de un programa de estudios provocado por factores que se generan tanto al interior del sistema educativo como por la sociedad, familia y entorno, considerando un tiempo suficiente para descartar la posibilidad de una reincorporación por parte del estudiante" (2) y se puede definir como "el proceso de abandono, voluntario o forzoso, del programa académico en el que se matriculó un estudiante" (3).

En América Latina, la deserción oscila entre el $40 \%$ y el 75\% (3) y según el Ministerio de Educación Nacional de Colombia (4), la tasa de deserción anual para el 2012 era del 11.1\%, ubicándolo entre los países intermedios con mayores avances en su sistema educativo. Según el Ministerio de Educación Nacional la meta para la tasa de deserción por periodo (anual) para la vigencia 2015 era $9.7 \%$ para el nivel universitario y se logró reducirla al 9.3\%; sin embargo, la tasa de deserción por cohorte en programas universitarios para 
ISSN-PRINT

1794-9831

E-ISSN 2322-7028

Vol. 16 No. 1

Ene - Abr 2019

Cúcuta, Colombia el 2015 fue del 46.1\% (5). Para Santander, en este mismo periodo la tasa de deserción universitaria fue del 39.6\% a corte de abril de 2016 (5). De acuerdo al Banco Mundial (6), "solo México y Perú tienen una tasa de finalización cercana a la de los Estados Unidos, es decir igual al $65 \%$ y para Colombia se estima que el $37 \%$ de los estudiantes que comienzan un programa de licenciatura abandonan el sistema educativo" (6), principalmente durante la primera mitad de la carrera; un $23.5 \%$ lo hace en el primer y segundo semestre y un 39\% en el cuarto semestre (7). En el caso del Programa de Enfermería de la Universidad Industrial de Santander según información de UIS en cifras para el año 2016, el retiro voluntario fue de $14.3 \%$.

En relación a las causas de la deserción, existen varios factores que inciden en ella (7-9), desde aspectos académicos hasta los individuales, económicos e institucionales. En Colombia el seguimiento a estos factores es desarrollado por el Sistema de Prevención de la Deserción en Educación Superior (SPADIES).

Este seguimiento se ha llevado a cabo desde el 2007 y sus resultados permiten conocer los determinantes de la deserción, lo cual posibilita en cada institución identificar y clasificar el riesgo de deserción (10) y diseñar estrategias para el abordaje del problema.

Los factores causales de la deserción analizados en el SPADIES son: socioeconómicos, individuales, académicos e institucionales (11). Los socioeconómicos son los más mencionados en los diferentes estudios sobre la deserción estudiantil (7,11-13), que incluyen aspectos del ámbito individual, familiar e institucional tales como: la relación, el apoyo y los rasgos del grupo familiar, la disponibilidad de recursos, valores del grupo familiar asociados al éxito, el ambiente familiar, bajos ingresos familiares, desempleo de los padres y con bajo nivel educativo, entre otros. Entre los factores individuales $(7,11)$, se encuentran aquellos aspectos asociados a la personalidad, la historia personal, las percepciones y actividades diarias del estudiante, así como a expectativas no satisfechas o que no le encuentran un futuro a lo que están estudiando (7).

Dentro de los factores académicos se encuentran (78, 14-16): la falta de preparación desde la educación media en competencias generales; la poca orientación profesional y vocacional antes del ingreso a la universidad; el bajo rendimiento académico; la baja calidad del programa al que se accede; los métodos de estudio y metodologías de aprendizaje obsoletas; la insatisfacción con el programa; el estrés por la carga académica.

Los factores institucionales, son $(1,7,11)$ : la falta de apoyos económicos por parte de la institución para matricula y sostenimiento; mala relación con los docentes y personal administrativo; bajo porcentaje de docentes por estudiante que afecta tanto la calidad, como el aprendizaje; e inestabilidad en el ritmo académico en las universidades públicas.

En concordancia con lo anterior, se ha propuesto un estudio para determinar los factores relacionados con la intención de desertar en estudiantes de enfermería de una universidad pública en Colombia.

Los objetivos específicos son identificar desde el ámbito individual, socioeconómico, académico e institucional los factores relacionados con la intención de desertar en estudiantes de enfermería y describir la frecuencia de la intención de deserción en la población de estudio, lo cual será insumo para el diseño de estrategias en la disminución de la deserción estudiantil, mediante el mejoramiento de los procesos académicos y administrativos que permitan formular políticas en la institución y a nivel nacional, dado que es un problema social.

\section{Materiales y métodos}

Estudio de corte transversal analítico en estudiantes de un programa de Enfermería de una universidad pública en Colombia.

Se realizó un muestreo no probabilístico. Se divulgó una convocatoria pública a través de la página web de la Universidad y carteleras dirigida a la totalidad de los estudiantes (203) del programa de Enfermería y se obtuvo una tasa de respuesta positiva del $79.8 \%$ $(n=162)$. Criterio de inclusión: estudiantes activos en el programa de enfermería de una universidad pública en Colombia para el segundo semestre del 2016. Criterio de exclusión: antecedente reciente (1 mes) de participación en actividades de investigación relacionadas con la deserción escolar.

Teniendo en cuenta la literatura científica e investigaciones previas relacionadas con el tema de interés al interior de la institución, se diseñó un cuestionario "Factores de riesgo de deserción estudiantil universitaria" con 55 ítems previamente explorados o identificados como factores relacionados con la deserción de la población universitaria, de los cuáles 46 eran preguntas 
cerradas (18 dicotómicos y 28 politómicos) y 9 fueron preguntas abiertas, distribuidas en los siguientes cuatro factores: I. factores individuales, II factores socioeconómicos, II factores académicos, y IV factores institucionales. En el factor individual se incluyó el instrumento Hopkins Symptom Checklist (HSCL-10) para clasificar a los estudiantes sintomático depresivos o ansiosos, de este instrumento se encuentran reportes de consistencia interna, alfa de Cronbach entre 0.86-0.89 (17). Una vez que el equipo investigador realizó la validez facial del instrumento e incorporó las mejores, se realizó la validez facial con la población objeto, para lo cual se hizo una selección aleatoria de 32 estudiantes de los diferentes niveles del programa, a quienes se les solicitó la evaluación de la comprensibilidad y claridad del instrumento. Los investigadores consolidaron los resultados e incorporaron los ajustes correspondientes.

A partir de la versión final del instrumento se creó un formulario en Google Docs, previo diligenciamiento online del mismo y se realizó proceso de consentimiento informado con cada uno de los participantes. Una vez finalizado el periodo de reclutamiento se generó una hoja de cálculo google, la cual, tras ser descargada, fue codificada y exportada para su análisis al programa estadístico Stata versión 12.0/SE.

Se realizó la descripción de la población a través de frecuencias absolutas y relativas para variables categóricas y medidas de tendencia central de acuerdo a la distribución de este tipo de variables, la cual se evaluó a través de la prueba Shapiro Wilk a un nivel de significancia de $p<0,005$. Las variables con distribución normal se mostraron con medias y desviación estándar (DE) y las que no cumplieron con esta condición se presentaron con medianas y rangos intercuartílicos. Los puntajes del instrumento Hopkins Symptom Checklist (HSCL-10) fueron procesados de acuerdo al punto de corte 1.85 sugerido en la literatura para clasificar a los adolescentes sintomáticos depresivos o ansiosos.

Asumiendo la intención de deserción de la carrera de enfermería como variable dependiente, se realizó el análisis bivariado (regresión binomial) con cada una de las variables independientes recolectadas en el cuestionario, algunas fueron reagrupadas debido a muy bajas frecuencias en alguna de sus categorías de respuesta, en estas variables se exploró colinealidad. Teniendo en cuenta que la prevalencia de la intención de deserción de la carrera de enfermería superó el 10\%, se construyó un modelo de regresión binomial para identificar los factores asociados a la intención de deserción de la carrera de enfermería. Al modelo se le incluyeron variables que en el análisis bivariado presentaron un valor de $\mathrm{p}<0,20$ y aquellas que tenían relevancia desde la literatura.

El modelo final debía cumplir con los siguientes requisitos: ser parsimonioso e incluir variables con significancia estadística $(\mathrm{p}<0.05)$. Se evaluó confusión por sexo y edad. Finalmente, se evaluó AUC ROC (área bajo la curva). Todos los análisis se realizaron en el programa estadístico Stata versión 12.0/SE y algunas gráficas en Excel Microsoft 2011 14.1.0 Mac.

Esta investigación fue aprobada por el comité de ética en investigación científica de la universidad, según acta No. 18 del 7 de octubre de 2016 y se basó en los lineamientos de la Resolución No. 008430 de 1993 del Ministerio de Salud de Colombia, clasificándose como una investigación sin riesgo. Se obtuvo consentimiento informado escrito por parte de los participantes garantizando el respeto, la autonomía y la confidencialidad en la información suministrada para la investigación.

\section{Resultados}

El promedio de edad fue 21 años (DE) \pm 2.5 , el $85 \%$ $(\mathrm{n}=137)$ pertenecen al género femenino, $94 \%(\mathrm{n}=153)$ tenían hermanos, $29 \%(\mathrm{n}=47)$ provenían de otros municipios, $13 \%(\mathrm{n}=21)$ residían con personas diferentes a su familia nuclear, en promedio reportaron dormir 5.5 horas/día (DE) \pm 1.2 . El 20\% ( $=32)$ declaró haber considerado desertar del programa de enfermería en el último mes, de estos 78\% (25/32) cursaban sexto o niveles inferiores. De igual forma, al indagar por las razones, se encontró que en términos de los estudiantes, éstas estaban mayormente relacionadas con motivos personales y vocacionales.

En cuanto a los hallazgos del factor individual, el $48 \%(\mathrm{n}=77)$ obtuvo un puntaje mayor a 1.85 en el HSCL-10, asimismo 19\% $(\mathrm{n}=12)$ reconoció que las relaciones con su familia eran regulares-malas, el $46.9 \%(\mathrm{n}=76)$ manifestó tener pareja, de los cuales el $7,8 \%(\mathrm{n}=6 / 76)$ consideró que la relación con su pareja era regular; por otra parte $27 \%(n=44)$ expresó que se cambiaría de carrera si tuviera la oportunidad, de estos el $66 \%(n=31 / 44)$ reportó que se cambiaría a medicina. En el análisis bivariado características como el género, tener pareja, relación con la pareja, familia y puntaje en el HSCL-10, entre otras, presentadas en 
ISSN-PRINT

1794-9831

E-ISSN 2322-7028

Vol. 16 No. 1

Ene - Abr 2019

Cúcuta, Colombia la tabla 1, mostraron relación con la intención de deserción.

En el factor socioeconómico se encontró que la distribución por estratos fue homogénea, además la mayoría reportó recibir apoyo económico total y el 78\% $(\mathrm{n}=121)$ señaló que el responsable del apoyo económico tenía un empleo estable. El 35\% (n=58) manifestó estar activo laboralmente, de estos, el $58 \%(n=34 / 58)$ trabaja 20 o más horas a la semana, esta variable mos- tró significancia en el análisis bivariado. Al indagar por los niveles de formación de los padres se encontró una mayor frecuencia de reporte de haber cursado algún grado de secundaria por parte de los papás $44 \%$ $(\mathrm{n}=72)$, en este nivel de formación las madres solo alcanzaron un $37 \%(n=60)$; contrario a lo anterior, en la formación técnica/tecnológica y universitaria fueron las madres quienes alcanzaron un $28 \%(\mathrm{n}=45)$ y $22 \%$ $(n=36)$, respectivamente de formación. (Tabla 1).

Tabla 1. Factores individuales y socioeconómicos relacionados con la intención de deserción según categorías reagrupadas para análisis bivariado

\begin{tabular}{|c|c|}
\hline Variable & $\%(n)$ \\
\hline \multicolumn{2}{|l|}{-Factores individuales } \\
\hline \multicolumn{2}{|l|}{ *Considera que su integración social es: } \\
\hline Regular & $24(39)$ \\
\hline Buena & $76(123)$ \\
\hline \multicolumn{2}{|l|}{ *Estado salud física últimos 6 meses } \\
\hline Regular-malo & $38(61)$ \\
\hline Bueno & $62(101)$ \\
\hline \multicolumn{2}{|c|}{ *Con respecto a su motivación personal para cursar esta carrera usted considera que es } \\
\hline Media-Baja & $36(59)$ \\
\hline Alta & $64(103)$ \\
\hline \multicolumn{2}{|c|}{$*_{¿ \text { Usted se ha proyectado como profesional de esta carrera? }}$} \\
\hline $\mathrm{Si}$ & $95(154)$ \\
\hline No & $5(8)$ \\
\hline \multicolumn{2}{|l|}{-Factores socioeconómicos } \\
\hline \multicolumn{2}{|l|}{ *Estrato socioeconómico } \\
\hline $1-2$ & $51(83)$ \\
\hline $3-4-5$ & $49(79)$ \\
\hline \multicolumn{2}{|l|}{ Apoyo económico } \\
\hline Total & $67(108)$ \\
\hline Parcial & $29(48)$ \\
\hline Ninguno & $4(6)$ \\
\hline
\end{tabular}

*factores con significancia estadística en el análisis bivariado $(\mathrm{p}<0.20)$

Fuente: Autoras. Datos segundo semestre 2016.

En relación al factor académico, el 83\% $(\mathrm{n}=135)$ realizó sus estudios de bachillerato en instituciones educativas oficiales y el porcentaje restante $17 \%(n=27)$ en privados. Al indagar por la percepción sobre el aporte de las asignaturas cursadas durante su secundaria para atender los objetivos académicos de la vida universitaria, el 71\% ( $\mathrm{n}=115)$ manifestó no percibir contribución. Por su parte, el 51,2\% (n=84) declaró haberse presentado a otra carrera de la universidad como primera elección, de los cuales el $85 \%(n=71 / 84)$ señaló que medicina fue su primera opción. También, se observó que el $51 \%(\mathrm{n}=83)$ reportó pérdida de al menos una asignatura y $94 \%(n=153)$ consideró interesantes las asignaturas del programa; 47\% $(n=76)$ reportó un promedio académico entre (3.7-3.9), seguido de un $32 \%(\mathrm{n}=52)$ en el rango de (3.3-3.6) y $20 \%(\mathrm{n}=33)$ un promedio entre (4.0-4.4). Asimismo, al solicitar que informaran en orden de importancia sobre las técnicas de estudio, en primer lugar, se referenció la lectura $63 \%$ (101/162), en segundo lugar, el resumen 31 (50/159) 
y revisión de los propios apuntes 30\% (45/153). En cuanto al factor institucional, el $77 \%(\mathrm{n}=125)$ pertenecía o había sido beneficiario de al menos un programa de bienestar estudiantil universitario. Cabe señalar, que adicional a las variables presentadas en la tabla 2, la satisfacción con el programa de enfermería también mostró relación con la intención de deserción.

Tabla 2. Factores académicos e institucionales relacionados con la intención de deserción según categorías reagrupadas para análisis bivariado

\begin{tabular}{|c|c|}
\hline Variable & $\%(n)$ \\
\hline \multicolumn{2}{|c|}{-Factores académicos } \\
\hline \multicolumn{2}{|c|}{ *Nivel matriculado } \\
\hline Segundo & $25(41)$ \\
\hline Cuarto & $23(38)$ \\
\hline Sexto & $12(19)$ \\
\hline Octavo & $27(44)$ \\
\hline Décimo & $20(12)$ \\
\hline \multicolumn{2}{|c|}{$*_{¿}$ Considera que los contenidos de las asignaturas recibidas en la secundaria lo prepararon para la Universidad? } \\
\hline $\mathrm{Si}$ & $29(47)$ \\
\hline No & $71(115)$ \\
\hline \multicolumn{2}{|c|}{ ¿Realizó estudios superiores previo ingreso al programa de enfermería? } \\
\hline $\mathrm{Si}$ & $21(34)$ \\
\hline No & $79(128)$ \\
\hline \multicolumn{2}{|c|}{ ¿Conocía el plan de estudios de la carrera de enfermería antes de postularse? } \\
\hline $\mathrm{Si}$ & $61(99)$ \\
\hline No & $39(63)$ \\
\hline \multicolumn{2}{|c|}{$*_{¿ \text { Considera interesante las asignaturas del programa académico? }}$} \\
\hline $\mathrm{Si}$ & $94(153)$ \\
\hline No & $6(9)$ \\
\hline \multicolumn{2}{|c|}{-Factores institucionales } \\
\hline \multicolumn{2}{|c|}{ *Relación con profesores } \\
\hline Buena & $95(154)$ \\
\hline Regular & $5(8)$ \\
\hline \multicolumn{2}{|c|}{ *Relación con compañeros } \\
\hline Buena & $89(145)$ \\
\hline Regular & $10(16)$ \\
\hline Mala & $1(1)$ \\
\hline \multicolumn{2}{|c|}{ * ¿Usted prefería estudiar en otra universidad? } \\
\hline $\mathrm{Si}$ & $2(4)$ \\
\hline No & $98(158)$ \\
\hline
\end{tabular}

*factores con significancia estadística en el análisis bivariado $(\mathrm{p}<0.20)$

Fuente: Las autoras. Datos segundo semestre 2016

\section{Análisis multivariado regresión binomial razón de prevalencias}

Se realizó la evaluación de la colinealidad a través del coeficiente de correlación de Spearman de las variables, que según el análisis bivariado debían incluirse en el modelo. Se encontraron correlaciones moderadas o fuertes
(Coeficiente de Spearman $\geq 0.4$ ) (18) con significancia estadística $\mathrm{p}<0.05$ por lo cual se redujo el número de variables a modelar. A continuación, se realizó un modelamiento stepwise forward (19) con las variables género, puntaje (dicotómico) HSCL-10, percepción, integración social en la universidad, relación con la familia, proyec- 
ISSN-PRINT

1794-9831

E-ISSN 2322-7028

Vol. 16 No. 1

Ene - Abr 2019

Cúcuta, Colombia ción como profesional de enfermería, estrato socioeconómico, interés por las asignaturas del programa de enfermería, relación con profesores, relación con la pareja, percepción de preparación en la secundaria para la vida universitaria y preferencia por estudiar en otra universidad. Se identificaron cinco sujetos con residuales marcadamente alejados de la tendencia general de los datos, finalmente se realizó el ajuste por género y edad.

Tabla 3. Factores asociados con la intención de deserción, modelo multivariado ajustado por género y edad $(\mathrm{n}=157)$

\begin{tabular}{lcccc}
\hline Variable & $\beta$ & RP & p & IC 95\% \\
\hline $\begin{array}{l}\text { Puntaje total Hopkins Symptomchecklist-10 } \\
(<1.85 \text { vs } \geq 1.85)\end{array}$ & 1.2 & 3.3 & 0.024 & $1.2 ; 9.3$ \\
$\begin{array}{l}\text { Estrato socioeconómico dicotómico } \\
\text { (estrato 5,4,3 vs estrato 1,2) }\end{array}$ & 1.4 & 4.1 & 0.008 & $1.4 ; 11.7$ \\
$\begin{array}{l}\text { ¿Usted encuentra interesantes las asignaturas del programa académico? } \\
\text { (Si vs No) }\end{array}$ & 3.4 & 29.0 & 0.002 & $3.5 ; 238.9$ \\
Relación con profesores (Buena vs Regular) & 2.2 & 8.7 & 0.031 & $1.2 ; 63.7$ \\
\hline
\end{tabular}

b: Beta; RP: Razón de Prevalencia; p=valor p; IC: Intervalo de Confianza

Fuente: Autoras. Datos segundo semestre 2016

En el modelo final la mayor relación de prevalencias se identificó con la variable interés por las asignaturas del programa de enfermería, identificándose que existe 29 veces más intención de deserción en el grupo que manifestó no sentir dicha afinidad por las asignaturas, que el grupo que afirmó estar interesa- do; otros factores como puntaje HSCL-10, estrato socioeconómico y relación con los profesores, mostraron asociación estadística con la intención de desertar de la carrera de enfermería (Tabla 3), el modelo final obtuvo un área bajo la curva de 0.80 (Figura 1).

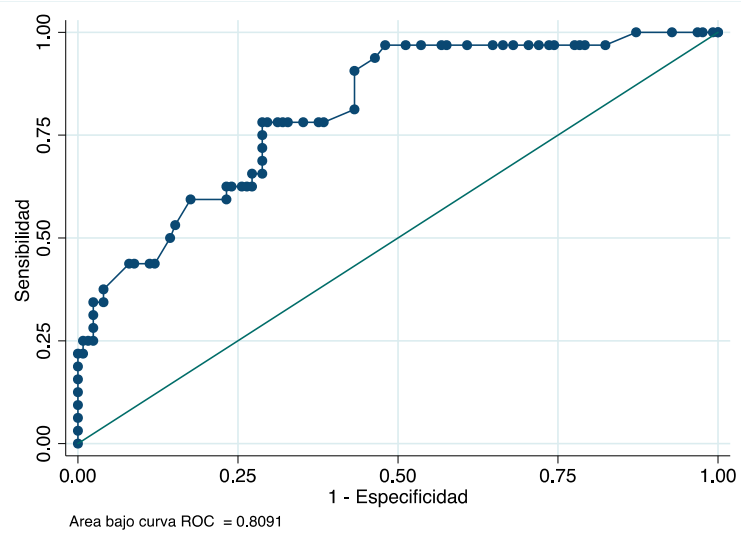

Figura 1. Curva ROC modelo final

Fuente: Autoras. Datos segundo semestre 2016

\section{Discusión}

La deserción estudiantil ha sido un tema de preocupación en los sistemas de educación superior en todo el mundo, debido a las implicaciones que esto tiene en los indicadores de desarrollo de un país, tanto en lo económico como en lo social y humano (20); pese a que algunos países se han esmerado por mejorar esta situación, el problema continúa, aunque para algunos las tasas de deserción son menores.

En Colombia al igual que en Latinoamérica, los sistemas de educación superior presentan históricamente altas tasas de deserción y aunque se ha mejorado en el seguimiento e investigación sobre el fenómeno (20), las tasas continúan altas; es así como para el 2015 se revela que para el nivel universitario esta fue del 
46.1\% por cohorte (21-22). El Ministerio de Educación espera disminuir esta tasa y se ha fijado disminuirla en un $25 \%$ para el 2019 , según el documento Visión Colombia II Centenario 2019 (20).

Existen una serie de factores que tienen relación con la deserción, los cuales han sido agrupados en categorías que abarcan desde los aspectos individuales hasta lo académico, institucional y socioeconómico. Al respecto, el Ministerio de Educación de Colombia ha implementado un sistema de seguimiento a esta problemática, lo cual permite conocer los factores de mayor incidencia en la deserción y a la vez diseñar estrategias de acuerdo a las particularidades de las instituciones. En este sentido, los resultados recientes del seguimiento han demostrado que el factor académico ha sido el principal causante del abandono de los estudios, seguido de los factores financieros, económicos, institucionales y de orientación vocacional y profesional respectivamente (20).

Dentro de los hallazgos generales de este estudio se encontró que los síntomas de ansiedad y depresión, la falta de interés en las asignaturas del programa, la percepción de regular relación con los profesores y pertenecer a estratos socioeconómico bajos, se asociaron con la intención de desertar de los estudiantes de enfermería. Al realizar el análisis se puede afirmar que cada uno de estos aspectos hace referencia de manera explícita a uno de los cuatro factores relacionados con la deserción estudiantil referidos en la literatura (20) y que fueron evidentes en este estudio.

Con relación al 48\% de estudiantes identificados como sintomáticos depresivos o ansiosos a través del HSCL-10 (23-24) y su correspondencia al factor individual, existe similitud con el hallazgo de AlFaris et al. (25), quienes diseñaron un estudio de corte transversal para determinar los síntomas depresivos en estudiantes de cuatro escuelas de salud de una universidad pública ubicada en Riyadh, Arabia Saudita, obteniendo una tasa general del $47 \%$ y del $51.6 \%$ para estudiantes de odontología, $46.2 \%$ para medicina, $45.7 \%$ para ciencias médicas aplicadas y $44.2 \%$ para enfermería. Asímismo, Cheung et al., (26) hallaron en su estudio en estudiantes de enfermería en Hong Kong una prevalencia de depresión leve a grave del $35.8 \%$, ansiedad $37.3 \%$ y de síntomas de estrés $41.1 \%$, respectivamente.

Como se observó en el modelo final, la mayor rela- ción de prevalencias se presentó con la variable "interés por las asignaturas del programa de enfermería", aspecto que está relacionado con el factor académico y el plan curricular, los cuales también han presentado relación con la decisión de los estudiantes de retirarse, como lo muestra, un estudio llevado a cabo por Ariza y Marín (27) en estudiantes desertores de un programa de psicología, que halló una alta correlación directa positiva $(\alpha 0.87)$ con el factor denominado "pérdida de interés por el programa elegido" y la deserción. Así mismo, Aburto (28) refiere que al indagar en los estudiantes acerca de los factores que influyeron para que se retiraran de la Universidad, el $57.8 \%$ indicaron que fue debido al plan de estudio y la metodología que utilizan los profesores. Además, el $31.6 \%$ consideraban que los programas estaban recargados de contenidos.

La relación regular con profesores como factor asociado a la intención de deserción, hallada en este estudio, es semejante con lo referido por Mori (29) quien indica que el trato con los docentes es una variable del factor institucional que puede incidir en la deserción. Igualmente, Aburto (28) describe que dentro de las principales causas que refirieron los estudiantes del estudio que desertaron estaba la "poca atención por parte de los profesores en el momento de que se les presentaron dificultades" con una frecuencia del 15\%. A su vez, Villamizar et al., (30) agregan que en los desertores del programa de Psicología se "observó de manera marcada y repetitiva que los problemas con los docentes intervinieron de manera significativa en ello".

En esta investigación fue un factor de riesgo de intención de deserción pertenecer a un estrato socioeconómico bajo, lo que implica menos recursos y dificultades para culminar los estudios. Este hallazgo coincide con lo encontrado por varios autores $(27,31-35)$. En este sentido, Gartner et al., (31-32) en estudios llevados a cabo en la Universidad de Caldas, Colombia, encontraron que los estudiantes que han abandonado sus estudios estaban ubicados en los estratos socioeconómicos más bajos.

De igual forma. Ariza (27) encontró que los "problemas económicos a nivel personal $\mathrm{y} / \mathrm{o}$ familiar" eran los aspectos más significativos y presentaban una alta correlación ( $\alpha$ 0.808) con la variable de deserción. Por el contrario, Restrepo (3) obtuvo un mayor porcentaje de deserción en los estratos 3 (47.2\%), seguido del 4 (27.8\%). De esta misma forma Barrero y colaboradores (36) señalaron 
ISSN-PRINT

1794-9831

E-ISSN 2322-7028

Vol. 16 No. 1

Ene - Abr 2019

Cúcuta, Colombia en su estudio que el factor económico tanto en mujeres como en hombres, fue el de menor importancia a la hora de tomar la decisión de retirarse de la carrera.

Es evidente que la deserción en la educación superior ha generado una gran preocupación, dado que conlleva a repercusiones en los estudiantes, la institución y la sociedad (37). Esto ha hecho que varias instituciones educativas indaguen sobre el tema (38-40) con el fin de precisar sobre los factores que inciden en la deserción estudiantil de manera particular en su organización. En este sentido, los resultados de este estudio han suministrado conocimientos sobre este problema, en un programa académico de una universidad pública, pero estos hallazgos pueden ser útiles para programas similares. Una de las limitaciones del presente estudio es solo contar con la intención de deserción, por lo cual se hace necesaria la realización de estudios longitudinales que permitan determinar el evento de desertar formalmente de un programa y evaluar al ingreso a la universidad posibles factores asociados a este hecho, facilitando el establecimiento de relaciones temporales entre las variables del estudio.

\section{Conclusiones}

- Los factores relacionados con la intención de desertar en estudiantes de enfermería son aca- démicos e individuales. Los factores académicos referidos fueron: bajo interés por asignaturas del programa y percepción de regulares relaciones con los profesores. A nivel individual se destacan la presencia de síntomas de depresión o ansiedad y pertenecer a bajos estratos socioeconómicos; estos 4 aspectos guardan relación con uno de los factores definidos para la deserción por el SPADIES. Lo anterior, es información importante tanto para el programa como para la institución educativa, puesto que permite priorizar acciones para intervenir la problemática.

- Los factores individuales de los estudiantes requieren mayor exploración, así como las habilidades de estudio.

- El 20\% de los estudiantes han considerado desertar del programa de enfermería en el último mes y se encontró que las razones en términos de los estudiantes estaban relacionadas con motivos personales y vocacionales.

\section{Conflicto de Intereses}

Los autores declaran no tener ningún conflicto de interés.

\section{Referencias bibliográficas}

1. Ministerio de Educación de Colombia, SPADIES, CEDE Facultad de Economía, Universidad de los Andes. Informe mensual sobre el soporte técnico y avance del contrato para garantizar la alimentación, consolidación, validación y uso de la información del SPADIES. Bogotá: Centro de Estudios sobre Desarrollo Económico, CEDE, Facultad de Economía, Universidad de los Andes; 2014.

2. Observatorio de Educación Superior de Medellín. Panorama internacional de la deserción en la educación superior. Boletín julio - 2017 N5. [Internet]. 2017 [consultado 22 de febrero de 2018]. Disponible en: http://www.sapiencia.gov.co/wp-content/uploads/2017/07/BOLETIN ODES DESERCION_EN_LA_EDUCACION_SUPERIOR.pdf

3. Restrepo-Vásquez A. Factores relacionados con la deserción estudiantil en el programa de enfermería de la Universidad Libre de Pereira. Cultura del Cuidado Enfermería. 2010; 7(2):5-14.

4. Ministerio de Educación Nacional. Subdirección de Desarrollo Sectorial. Acuerdo nacional para disminuir la deserción en educación superior [Internet]. 2014 [consultado 19 de febrero de 2018]; Disponible en: https://www.scribd.com/document/368898759/ministerio-politicas-nacional-disminuirdesercion-2012-pdf

5. Ministerio de Educación Nacional. Estadísticas deserción y graduación 2015. [Internet]. 2015 [consultado 14 de octubre de 2018]. Disponible en: https://www.mineducacion.gov.co/sistemasdeinformacion/1735/articles-357549_recurso_3.pdf

6. Ferreyra MM, Avitabile C, Botero J, Haimovich F, Urzúa S. At a Crossroads Higher Education in 
Latin America and the Caribbean. 2017 International Bank for Reconstruction and Development / The World Bank Washington. [Internet]. 2017 [consultado 19 de febrero de 2018]; Disponible en: http:// dx.doi.org/10.1596/978-1-4648-1014-5

7. Vries WD, León-Arenas P, Romero-Muñoz JF, Hernández Saldaña I. ¿Desertores o decepcionados? Distintas causas para abandonar los estudios universitarios. Revista de la Educación Superior. [Internet]. 2011 [consultado 28 de marzo de 2018]; 40(4):29-49. Disponible en: http://publicaciones.anuies. mx/pdfs/revista/Revista160_S1A3ES.pdf

8. Vergara Morales JR, Boj del Val E, Barriga O, Díaz Larenas C. Factores explicativos de la deserción de estudiantes de pedagogía. Rev. complut. educ. [Internet]. 2017 [consultado 27 de marzo de 2018]; 28(2): 609-630. Disponible en: http://dx.doi.org/10.5209/rev_RCED.2017.v28.n2.50009

9. Huesca-Ramírez MG, Castaño-Corvo MB. Causas de deserción de alumnos de primeros semestres de una universidad privada. Revista Mexicana de Orientación Educativa. [Internet]. 2007 [consultado 27 de marzo de 2018]; 5(12):34-39. Disponible en: http:/www.alfaguia.org/alfaguia/ files $/ 1319582164$ causas\%20de\%20desercion\%20en\%20una\%20universidad\%20privada.pdf

10. Suárez Montes N, Díaz Subieta LB. Estrés académico, deserción y estrategias de retención de estudiantes en la educación superior. Revista de Salud Pública [Internet]. 2015 [Consultado 20 octubre de 2018]; 17:300-13. Disponible en: http://dx.doi.org/10.15446/rsap.v17n2.52891

11. Cely Atuesta DC, Durán Gamba MG. Causas asociadas a la deserción estudiantil y estrategias de acompañamiento para la permanencia estudiantil. IV conferencia latinoamericana sobre el abandono de la educación superior, Medellin, Colombia. [Internet]. 2014 [Consultado 14 octubre de 2018]; Disponible en: http://www.alfaguia.org/www-alfa/images/PonenciasClabes/5/ponencia_99.pdf

12. González Ramírez T, Pedraza Navarro I. Variables sociofamiliares asociadas al abandono de los estudios universitarios. Educatio Siglo XXI [Internet]. 2017 [Consultado 14 octubre de 2018]; 35, N. ${ }^{\circ} 2$ : 365-87. Disponible en: http://dx.doi.org/10.6018/j/298651

13. Celis Schneider R, Flores Ramírez CL, Reyes Martínez MC, Venegas Villanueva H. Factores de riesgo de deserción presentes en alumnos repitentes de las carreras de enfermería y kinesiología en una universidad chilena. Ciencia y enfermería [Internet]. 2013 [Consultado 14 octubre de 2018]; 19(3):63-71. Disponible en: http://dx.doi.org/10.4067/S0717-95532013000300007

14. Zarate Rueda R, Mantilla Pinilla E. La deserción estudiantil UIS, una mirada desde la responsabilidad social universitaria. Zona próxima: revista del Instituto de Estudios Superiores en Educación [Internet]. 2014 [Consultado 18 octubre de 2018]; 21:120-33. Disponible en: https://dialnet.unirioja.es/ servlet/articulo? codigo $=6416680$

15. Isaza Restrepo A, Enríquez Guerrero C, Pérez Olmos I. Deserción y rezago académico en el programa de medicina de la Universidad del Rosario, Bogotá, Colombia. Rev Cienc Salud. [Internet]. 2016 [Consultado 18 octubre de 2018]; 14(2):231-45. Disponible en: https://dialnet.unirioja.es/servlet/articulo? codigo $=5599053$

16. Yepes Delgado FL, Beltrán Salazar M, Martínez Ceferino EY. Academic dropout among undergraduate students from Universidad de Antioquia School of Dentistry, 2004-2014. Rev Fac Odontol Univ Antioq. [Internet]. 2017 [Consultado 18 octubre de 2018]; 29(1):76-95. Disponible en: http://dx.doi. org/10.17533/udea.rfo.v29n1a5

17. Kleppang AL, Hagquist C. The psychometric properties of the Hopkins Symptom Checklist-10: a Rasch analysis based on adolescent data from Norway. Family practice [Internet]. 2016 [consultado el 16 de octubre de 2018]; 33(6):740-5. Disponible en: https://doi.org/10.1093/fampra/cmw091

18. Winter J, Cosling SD, Potter J. Comparing the Pearson and Spearman Correlation Coefficients Across Distributions and Sample Sizes: A Tutorial Using Simulations and Empirical Data. Psychol Methods. [Internet]. 2016 [Consultado 20 octubre de 2018]; 21(3):273-90. Disponible en: http://dx.doi. org $/ 10.1037 /$ met0000079
E-ISSN 2322-7028

Vol. 16 No. 1

Ene - Abr 2019

Cúcuta, Colombia 
ISSN-PRINT

1794-9831

E-ISSN 2322-7028

Vol. 16 No. 1

Ene - Abr 2019

Cúcuta, Colombia

19. Menard S. Applied logistic regression analysis. [libro en internet]. 2002; Sage publications. [consultado 27 de marzo de 2018]; Disponible en: https://uk.sagepub.com/en-gb/eur/applied-logistic-regression-analysis/book11277

20. Ministerio de Educación Nacional, Colombia. Deserción estudiantil en la educación superior colombiana. Metodología de seguimiento, diagnóstico y elementos para su prevención. [Internet]. 2009 [consultado 21 de marzo de 2018]. Disponible en: https://www.mineducacion.gov.co/sistemasdeinformacion/1735/articles-254702 libro_desercion.pdf

21. Melo-Becerra LA, Ramos-Forero, JE, Hernández-Santamaría, PO. La educación superior en Colombia: situación actual y análisis de eficiencia. Revista Desarrollo y Sociedad. [Internet]. 2017 [consultado 27 de marzo de 2018]; 78:59-111. Disponible en: http://www.scielo.org.co/pdf/dys/n78/n78a03.pdf

22. Orozco Silva, LE. Educación superior en Iberoamérica informe 2016. Informe Nacional: Colombia. [Internet]. 2016 [consultado el 27 de marzo de 2018]; Disponible en: https://www.cinda.cl/2016/11/16/ informe-educacion-superior-en-iberoamerica-2016/

23. Rikard-Haavet O, Kaur-Sirpal M, Haugen W, Sparle-Christensen K. Diagnosis of depressed young people in primary health care. Family Practice. [Internet]. 2011 [consultado el 27 de marzo de 2018]; 28 (Issue 2): 233-237. Disponible en: https://doi.org/10.1093/fampra/cmq078

24. AlFaris E, Irfan F, Qureshi R, Naeem N, Alshomrani A, Ponnamperuma G, Yousufi N Al, Maflehi $\mathrm{N}$ Al, Naami M Al, Jamal A y Vleuten C. Health professions' students have an alarming prevalence of depressive symptoms: exploration of the associated factors BMC Medical Education [Internet]. 2016 [consultado el 27 de marzo de 2018]; 16:279. Disponible en: https://doi.org/10.1186/s12909016-0794-y

25. Cheung T, Wong S, Wong K, Law L, Ng K et al. Depression, Anxiety and Symptoms of Stress among Baccalaureate Nursing Students in Hong Kong: A Cross-Sectional Study. Int. J. Environ. Res. Public Health. [Internet]. 2016 [consultado el 27 de marzo de 2018]; 13(8), 779 Disponible en: https://doi. org/10.3390/ijerph13080779

26. Ariza Gasca SM, Marín-Arias DA. Factores intervinientes en la deserción escolar de la Facultad de Psicología. [Tesis internet]. Fundación Universitaria Los Libertadores. 2009. Bogotá. [consultado el marzo 27 de marzo 03 de 2018]; Disponible en: http://www.alfaguia.org/alfaguia/files/1320349840_35. pdf

27. Aburto-Jarquín P. Deserción estudiantil universitaria UNAN Managua, Farem Carazo. Revista Torreón Universitario. [Internet]. 2013 [consultado el 27 de marzo de 2018]; (5): 1-10. Disponible en: http://repositorio.unan.edu.ni/5874/1/75-238-1-PB.pdf

28. Mori-Sánchez MP. Deserción universitaria en estudiantes de una universidad privada de Iquitos. Revista Digital de Investigación en Docencia Universitaria. [Internet]. 2012 [consultado el 27 de marzo de 2018]; 6 (1): 60-83. Disponible en: http://revistas.upc.edu.pe/index.php/docencia/article/view/42/11

29. Villamizar Acevedo GA, Pérez-Bayona L. Identificación de factores motivacionales y sociodemográficos de estudiantes desertores de la Facultad de Psicología de la Universidad Pontificia Bolivariana Bucaramanga. Revista Psicogente [Internet]. 2011[consultado el 27 de marzo de 2018]; 14 (25): 13250. Disponible en: http://www.redalyc.org/pdf/4975/497552358012.pdf

30. Gartner Isaza L, Dussán Lubert C, Montoya-Londoño DM. Caracterización de la deserción estudiantil en la Universidad de Caldas el período 2009-2013. Análisis a partir del Sistema para la Prevención de la Deserción de la Educación Superior -SPADIES. Revista latinoam.estud.educ. [Internet]. 2016 [consultado el 20 de marzo de 2018]; 12(1):132-158. Disponible en: http://www.redalyc.org/ html/1341/134149742008/

31. Gartner Isaza L, Dussán Lubert C, Montoya-Londoño DM. Aproximación causal al estudio de la deserción en la Universidad de Caldas. Periodo 2012-2014. Revista Colombiana de Educación. [Internet]. 2016 [consultado 27 de marzo de 2018]; 70:319-340. Disponible en: http://www.scielo.org.co/ 
pdf/rcde/n70/n70a15.pdf

32. Heredia Alarcón M, Andía Ticona M, Ocampo Guabloche H, Ramos Castillo J, Rodríguez Caldas A, Tenorio C, et al. Deserción estudiantil en las carreras de ciencias de la salud en el Perú. An Fac Med. [Internet]. 2015 [consultado el 30 de marzo de 2018]; 76:57-61. Disponible en: http://revistasinvestigacion.unmsm.edu.pe/index.php/anales/article/view/10972/9904.

33. Parada Rico DA, Correa Suárez LY, Cárdenas Gonzáles YF. Factores relacionados con la permanencia estudiantil en programas de pregrado de una universidad pública. Investig Enferm. Imagen y Desarr [Internet]. 2017[consultado el 10 de marzo de 2018]; 19(1):155-170. Disponible en: http:/www.redalyc.org/html/1452/145249416011/

34. Londoño Ardila LF. Factores de riesgo presentes en la deserción estudiantil en la Corporación Universitaria Lasallista. Revista virtual Universidad Católica del Norte [Internet]. 2013 [consultado 27 de marzo de 2018]; 38:183-194. Disponible en: http://www.redalyc.org/articulo.oa?id=194225730014.

35. Barrero Rivera F, Barrero Espinosa C, Borja Cely H. Factores de riesgo asociados a la deserción estudiantil universitaria en programas de pregrado de la Universidad de San Buenaventura, Bogotá (20092013). Revista Academia y Virtualidad [Internet]. 2015 [consultado 27 de marzo de 2018]; 8(2):60-72. Disponible en: http://virtual2.umng.edu.co/distancia/revista/articulos/133.pdf

36. Sánchez Hernández G, Barboza Palomino M, Castilla-Cabello H. Análisis de la deserción y los factores asociados a la permanencia estudiantil en una universidad peruana. Actual. Pedagog. [Internet]. 2017 [consultado 29 de marzo de 2018]; 69:169-91. Disponible en: https://revistas.lasalle.edu.co/index.php/ap/article/view/4075

37. Universidad Industrial de Santander, Vicerrectoría Académica. Diagnóstico de las causas de deserción y retención estudiantil en los programas de pregrado presencial de la Universidad Industrial de Santander. [Internet]. 2011 [consultado 14 de noviembre de 2018]. Disponible en: https://vdocuments. $\mathrm{mx} /$ diagnostico-de-las-causas-de-desercion-y-retencion-estudiantil-en-.html

38. Rojas Betancur M. La deserción estudiantil en la Universidad de Ibagué: la perspectiva de los "desertores". Revista Virtual Universidad Católica del Norte [Internet]. 2008 [consultado el 26 de marzo de 2018]; 25. Disponible en: http://revistavirtual.ucn.edu.co/index.php/RevistaUCN/article/viewFile/123/240

39. Bravo F, Illescas L, Larriva S, Peña M. Causas de Deserción en el Ingreso a la Universidad; un Estudio de Caso. Revista de la Facultad de Ciencias Químicas [Internet]. 2017 [consultado el 29 de marzo de 2018]; 18:48-59. Disponible en: https://publicaciones.ucuenca.edu.ec/ojs/index.php/quimica/article/ view/1693/1316

40. Valladão M, Pomeroy D, Salgado A. Estudio de un caso: la deserción de estudiantes de primer año en la Escuela de Química de la Universidad Federal de Río de Janeiro (UFRJ). Av. cien. ing. [Internet]. 2013 [consultado el 30 de marzo de 2018]; 4(3):63-72. Disponible en: https://dialnet.unirioja.es/servlet/articulo? codigo $=4685983$ 